
\title{
$\begin{array}{ll}\text { Research Square } & \begin{array}{l}\text { Preprints are preliminary reports that have not undergone peer review. } \\ \text { They should not be considered conclusive, used to inform clinical practice, } \\ \text { or referenced by the media as validated information. }\end{array}\end{array}$
}

\section{Association of Body Image and Body Mass Index with psychological outcomes in obese patients.}

Virginia Campedelli

Sapienza University of Rome: Universita degli Studi di Roma La Sapienza

Chiara Ciacchella

Sapienza University of Rome: Universita degli Studi di Roma La Sapienza

Giorgio Veneziani

Sapienza University of Rome: Universita degli Studi di Roma La Sapienza

Irene Meniconzi

Sapienza University of Rome: Universita degli Studi di Roma La Sapienza

Emanuela Paone

Sapienza University of Rome: Universita degli Studi di Roma La Sapienza

Gianfranco Silecchia

Sapienza University of Rome: Universita degli Studi di Roma La Sapienza

Carlo Lai ( $\square$ carlo.lai@uniroma1.it)

Universita degli Studi di Roma La Sapienza https://orcid.org/0000-0002-7638-0375

\section{Research Article}

Keywords: body image, hopelessness, psychophysical well-being, bariatric surgery, obesity

Posted Date: January 5th, 2022

DOI: https://doi.org/10.21203/rs.3.rs-1207587/v1

License: (c) (i) This work is licensed under a Creative Commons Attribution 4.0 International License. Read Full License 


\section{Abstract}

Purpose

The purpose of this study was to test whether higher dissatisfaction with one's own body image, majorly than the body mass index (BMI), may be associated with higher levels of psychopathological aspects, hopelessness feelings, and psychophysical health.

Methods

Fifty-nine patients undergoing bariatric surgery filled out the Symptom Checklist-90-Revised, the Body Uneasiness Test, the 12-item Short Form Survey, the Beck Inventory Scale II, and the Beck Hopelessness Scale. Correlations and multiple regressions between measures were performed.

Results

Dissatisfaction with the perception of one's own body image was strongly correlated with a worse psychophysiological health. On the contrary, BMI showed no significant correlation with the previous variables. Furthermore, the perception of one's own body image significantly predicted the state of psychological health.

Conclusion

The findings of the present study showed a more relevant role of body image compared to the BMI in the association with psychological outcomes suggesting the importance to consider body image in the assessment and treatment of obese patients.

Level of evidence

level II, evidence obtained from well-designed controlled trials without randomization.

\section{Introduction}

Bariatric surgery is currently the gold standard for the treatment of morbid obesity and its comorbidities, leading to an improvement in psychophysical wellbeing [1]. Concerns about body image can significantly affect an individual with obesity and it is expected to have a negative clinical impact influencing their behaviour, quality of life and psychological health [2]. These can be some of the main motivations for weight loss in individuals with obesity [3] and a factor that motivates the decision for in treatment-seeking.

Many previous studies investigated the association between the weight and the psychological aspects in obese patients. Most of the studies showed a correlation with psychological aspects as anxiety, depression, low self-confidence, alexithymia, and eating disorders in overweight and obese patients [4-8].

Furthermore, the literature focuses on the perception of one's own body image after bariatric surgery, reporting different results. One recent study [9] examined psychological well-being and body image in a sample of pre-bariatric surgery patients and in those with 1-year follow-up, showing a body image and psychological well-being improved in most patients. In general, obese patients pre or post bariatric surgery showed a clear association between body image perception and psychological state [10-13].

Instead, a recent review [14] on the goal of examining body image changes in patients with obesity pre and post bariatric surgery, shows that in most cases the perception of one's body image does not change between pre and post bariatric surgery suggesting that body image is not directly associated with the effective weight in obese patients. This could be due to a difficulty to adapt the own body image to the physical change due to the surgical intervention. Other studies reveal how obese patients can suffer from psychiatric diseases such as depression and anxiety due to a severe dissatisfaction with their body image, even after bariatric surgery $[10,11]$. Furthermore, previous studies showed how body dissatisfaction seems to be correlated with hopelessness feeling in patients with normal weight [15].

The relevance of the body image is also showed by the paradox of post bariatric surgery patients where body image does not change after bariatric surgery [14]. Nevertheless, the reasons for the maintenance of an obese identity and dissatisfaction about body image, despite the weight loss, is still unclear and the studies existing in literature show the complexity of this issue (Ibidem).

The aim of this study is to investigate the association between the perception of one's own body image in relation to aspects of psychophysical health in a preliminary phase of bariatric surgery. In particular, the hypothesis of this study is that a higher dissatisfaction with one's own body image, majorly than the body mass index (BMI), may be associated with higher levels of psychopathological aspects and hopelessness feelings, together with lower levels of psychophysical health.

\section{Materials And Methods}

\section{Procedures}

This study was conducted at the Bariatric Centre of Excellence IFSO-EC of "La Sapienza" University of Rome, in collaboration with the Department of Dynamic, Clinical Psychology and Health Studies of "La Sapienza” University of Rome. 
The participants were recruited among patients attending the psychological assessment for bariatric surgery eligibility in the Bariatric Centre of Excellence IFSO-EC of "La Sapienza" University of Roma, between November 2020 and July 2021. Informed consent was obtained from all individual participants included in the study. The recruitment has been carried out by a trained clinical psychologist one month before surgery and the patients completed the selfreported anonymous questionnaires alone in a private room.

The exclusion criteria were (a) not being Italian citizen; (b) presence of psychopathology; (c) presence of drugs or alcohol abuse; (d) Ineligibility for surgery according to the European Guidelines on Metabolic and Bariatric Surgery [16]; (e) undergoing primary bariatric procedures. The exclusion criteria were evaluated during the preoperative psychological assessment.

This study was performed in line with the principles of the Declaration of Helsinki. Approval was granted by the Ethics Committee of University Department of Dynamic, Clinical Psychology and Health Studies of “La Sapienza” University of Rome (11/27/20, No 0001118).

\section{Measures}

Patients underwent an interview conducted by the clinical research psychologist where they were asked socio-demographic information such as gender, age, weight, hight and BMI, marital status, education level and work status. The questionnaires administered were the following.

The Symptom Checklist-90-Revised (SCL-90-R) [17] is a widely used checklist that measures levels of psychological symptoms experienced in the past week, on a five-point Likert scale ranging from "not at all" to "extremely." The global measures are the Global Severity Index (Global Severity Index), the Positive Symptom Total (PST) and the Positive Symptom Distress Index (PSDI). In addition, there are nine subscales describing different psychological aspects. The Italian version was used [18] and the reliability coefficients of internal consistency were good for this study (Cronbach's alpha values $=.76-.87$ ).

The Body Uneasiness Test (BUT) [19] is a self-administered questionnaire for the clinical assessment of body image disorders and related psychopathologies. It consists in two parts: BUT-A is a 34-item scale which indicates the presence of body uneasiness, and its' global measure is the Global Severity Index (BUT-A GSI) and, in addition, there are five subscale scores regarding specifics uneasiness on body image; the BUT-B is a 37-item scale assessing specific worries about body parts, shapes, or functions. The global measures are the Positive Symptom Total (BUT-PST), and the Positive Symptom Distress Index (PSDI). Also, the eight subscales subdivide different parts of the body. The validity of BUT demonstrated good internal consistency (BUT-A Cronbach's a values=.57-.88; BUT-B Cronbach's a values $=.64-.88$ )

The 12-item Short Form Survey (SF-12) [20] is a generic quality of life (QoL) instrument that includes a subset of 12 items from the 36-item Short Form Survey. The global measure is Total Score (SF-12 Total), with the subscales of Physical Health and Mental Health. Higher scores indicate an improvement in QoL. For this study, the Italian version was used [21] and the internal consistency reliability coefficients were good (Cronbach's a value $=-.63)$.

The Beck Inventory Scale II (BDI II) [22] assesses the presence and severity of depressive symptoms. A total score above 10 is the beginning of the presence of depression. High scores show greater severity of depression. For this study, the Italian version was used [23] and it showed good internal consistency with a Cronbach coefficient a of .84 .

The Beck Hopelessness Scale (BHS) [24] assesses the presence of hopelessness and negative expectations about the future, indicating the risk of suicide. It consists of 20 items in the form of yes-no questions and higher scores indicate more severe despair. For this study, the Italian version was used [25] and internal consistency was good with a Cronbach's a of .73 .

\section{Statistical analyses}

Continuous variables (age, weight, SCL-90-R, BUT, SF-12, BDI II and BHS) are presented as mean \pm standard deviation, counts or percentages. Categoric variables (sex, BMI, education level, work, and marital status) are presented as counts and percentages.

Correlational analyses (Pearson's r) between weight, BMI, BUT and the psychological outcomes were performed. Moreover, multiple regression analyses were performed including BMI, BUT-A Global Severity Index and BUT-B Positive Symptom Distress Index as predictors of psychological outcomes.

All analyses were performed with STATISTICA 8.0 software (StatSoft Inc.) and criterion for statistical significance was $p<0.05$.

\section{Results}

A total of 59 participants were enrolled between November 2020 and July 2021 . They were predominately female $(n=44 ; 74.6 \%)$ and the average age was 39.17 years $(S D=10.11$; range $19-55)$. Table 1 shows the participants' socio-demographic and psychological characteristics. 
Table 1

Socio-demographic and psychological characteristics of participants $(n=59)$.

\begin{tabular}{|c|c|c|c|}
\hline & Mean & SD & $\begin{array}{l}N(\%) \\
(n=59)\end{array}$ \\
\hline Weight & 115.62 & 22.89 & \\
\hline BMI baseline & 41.96 & 5.38 & $6(10.2 \%)$ \\
\hline Class I(30.0-34.9) & & & $15(25.4 \%)$ \\
\hline Class II(35.0-39.9) & & & $38(64.4 \%)$ \\
\hline \multicolumn{4}{|l|}{ Class III(>40.0) } \\
\hline Education & & & $35(59.3 \%)$ \\
\hline Primary School & & & $17(28.9 \%)$ \\
\hline Secondary School & & & $7(11.8 \%)$ \\
\hline \multicolumn{4}{|l|}{ University } \\
\hline Work & & & $4(6.9 \%)$ \\
\hline Student & & & $28(47.3 \%)$ \\
\hline Employed & & & $26(44.1 \%)$ \\
\hline Unemployed & & & $1(1.7 \%)$ \\
\hline \multicolumn{4}{|l|}{ Retired } \\
\hline Marital Status & & & $20(33.9 \%)$ \\
\hline Single & & & $32(54.2 \%)$ \\
\hline Married & & & $6(10.2 \%)$ \\
\hline Separated & & & $1(1.7 \%)$ \\
\hline \multicolumn{4}{|l|}{ Widowed } \\
\hline SCL-90-R & 43.66 & 1.09 & $5(8.5 \%)$ \\
\hline SCL- 90-R Global Severity Index & 49.81 & 2.72 & $14(23.7 \%)$ \\
\hline $\mathrm{T}<45$ & 60.17 & 2.70 & $15(25.4 \%)$ \\
\hline $45 \leq \mathrm{T}<55$ & 83.18 & 13.10 & $25(42.4 \%)$ \\
\hline $55 \leq T<65$ & 43.35 & 1.54 & $7(11.9 \%)$ \\
\hline$T \geq 65$ & 50.31 & 3.13 & $17(28.8 \%)$ \\
\hline SCL-90-R Positive Symptom Total & 60.05 & 2.85 & $13(22.0 \%)$ \\
\hline $\mathrm{T}<45$ & 74.04 & 5.95 & $22(37.3 \%)$ \\
\hline $45 \leq T<55$ & 43.10 & 1.25 & $3(5.1 \%)$ \\
\hline $55 \leq T<65$ & 49.83 & 3.24 & $8(13.5 \%)$ \\
\hline$T \geq 65$ & 59.69 & 2.43 & $23(39.0 \%)$ \\
\hline SCL-90-R Positive Symptom Distress Index & 76.37 & 12.33 & $25(42.4 \%)$ \\
\hline $\mathrm{T}<45$ & 42.09 & 1.02 & $6(10.2 \%)$ \\
\hline $45 \leq \mathrm{T}<55$ & 50.16 & 2.84 & $10(17.0 \%)$ \\
\hline $55 \leq T<65$ & 58.38 & 2.24 & $11(18.6 \%)$ \\
\hline$T \geq 65$ & 81.79 & 14.29 & $32(54.2 \%)$ \\
\hline SCL-90-R Somatisation & 43.11 & 0.99 & $5(8.5 \%)$ \\
\hline $\mathrm{T}<45$ & 50.10 & 3.57 & $20(33.9 \%)$ \\
\hline $45 \leq T<55$ & 60.78 & 2.70 & $12(20.4 \%)$ \\
\hline $55 \leq T<65$ & 78.30 & 13.86 & $22(37.2 \%)$ \\
\hline$T \geq 65$ & 42.56 & 0.00 & $6(10.2 \%)$ \\
\hline SCL-90-R Obsessiveness-Compulsiveness & 50.43 & 3.36 & $21(35.5 \%)$ \\
\hline $\mathrm{T}<45$ & 60.47 & 2.15 & $7(11.9 \%)$ \\
\hline
\end{tabular}

Page 4/13 


\begin{tabular}{|c|c|c|c|}
\hline $45 \leq T<55$ & Rieân & SD 62 & $25(42.4 \%)$ \\
\hline $55 \leq \mathrm{T}<65$ & 42.69 & 1.01 & $(4=699)$ \\
\hline$T \geq 65$ & 48.44 & 2.77 & $19(32.2 \%)$ \\
\hline SCL-90-R Interpersonal Hypersensitivity & 57.55 & 2.29 & $8(13.6 \%)$ \\
\hline $\mathrm{T}<45$ & 76.97 & 8.53 & $28(47.3 \%)$ \\
\hline $45 \leq T<55$ & 43.24 & 1.41 & $12(20.3 \%)$ \\
\hline $55 \leq \mathrm{T}<65$ & 49.29 & 1.77 & $19(32.2 \%)$ \\
\hline$T \geq 65$ & 58.11 & 2.21 & $10(17.0 \%)$ \\
\hline SCL-90-R Depression & 79.73 & 8.03 & $18(30.5 \%)$ \\
\hline $\mathrm{T}<45$ & 41.89 & 0.00 & $18(30.5 \%)$ \\
\hline $45 \leq \mathrm{T}<55$ & 48.27 & 2.32 & $12(20.4 \%)$ \\
\hline $55 \leq \mathrm{T}<65$ & 60.21 & 3.98 & $15(25.4 \%)$ \\
\hline$T \geq 65$ & 83.08 & 16.37 & $14(23.7 \%)$ \\
\hline SCL-90-R Anxiety & 41.89 & 0.00 & $23(39.0 \%)$ \\
\hline $\mathrm{T}<45$ & 48.44 & 3.17 & $23(39.0 \%)$ \\
\hline $45 \leq \mathrm{T}<55$ & 58.44 & 1.88 & $7(11.9 \%)$ \\
\hline $55 \leq T<65$ & 74.07 & 12.61 & $6(10.2 \%)$ \\
\hline$T \geq 65$ & 42.27 & 0.00 & $12(20.4 \%)$ \\
\hline SCL-90-R Hostility & 50.39 & 3.27 & $14(23.7 \%)$ \\
\hline $\mathrm{T}<45$ & 59.11 & 2.00 & $9(15.2 \%)$ \\
\hline $45 \leq \mathrm{T}<55$ & 84.10 & 18.94 & $24(40.7 \%)$ \\
\hline $55 \leq \mathrm{T}<65$ & 44.40 & 0.00 & $17(28.8 \%)$ \\
\hline$T \geq 65$ & 49.47 & 1.83 & $15(25.4 \%)$ \\
\hline SCL-90-R Phobic Anxiety & 61.20 & 3.16 & $10(17.0 \%)$ \\
\hline $\mathrm{T}<45$ & 85.81 & 14.96 & $17(28.8 \%)$ \\
\hline \multicolumn{4}{|l|}{$45 \leq \mathrm{T}<55$} \\
\hline \multicolumn{4}{|l|}{$55 \leq T<65$} \\
\hline \multicolumn{4}{|l|}{$T \geq 65$} \\
\hline \multicolumn{4}{|l|}{ SCL-90-R Paranoid Ideation } \\
\hline \multicolumn{4}{|l|}{$\mathrm{T}<45$} \\
\hline \multicolumn{4}{|l|}{$45 \leq T<55$} \\
\hline \multicolumn{4}{|l|}{$55 \leq T<65$} \\
\hline \multicolumn{4}{|l|}{$T \geq 65$} \\
\hline \multicolumn{4}{|l|}{ SCL-90-R Psychoticism } \\
\hline \multicolumn{4}{|l|}{$\mathrm{T}<45$} \\
\hline \multicolumn{4}{|l|}{$45 \leq T<55$} \\
\hline \multicolumn{4}{|l|}{$55 \leq T<65$} \\
\hline$T \geq 65$ & & & \\
\hline
\end{tabular}




\begin{tabular}{|c|c|c|c|}
\hline & Mean & SD & $\begin{array}{l}N(\%) \\
(n=59)\end{array}$ \\
\hline BUT-A & 0.72 & 0.35 & $18(30.5 \%)$ \\
\hline BUT-A Global Severity Index & 2.44 & 0.75 & $41(69.5 \%)$ \\
\hline Score $<1.2$ & 1.66 & 0.84 & $40(67.8 \%)$ \\
\hline Score $>1.2$ & 3.73 & 0.64 & $19(32.2 \%)$ \\
\hline BUT-A Weight Phobia & 1.65 & 0.82 & $27(45.8 \%)$ \\
\hline Score $0.00-2.99$ & 3.86 & 0.46 & $32(54.2 \%)$ \\
\hline Score $3.00-5.00$ & 1.00 & 0.84 & $51(86.4 \%)$ \\
\hline BUT-A Body Image Concern & 3.63 & 0.67 & $8(13.6 \%)$ \\
\hline Score 0.00-2.99 & 0.85 & 0.67 & $56(94.9 \%)$ \\
\hline Score $3.00-5.00$ & 3.22 & 0.10 & $3(5.1 \%)$ \\
\hline BUT-A Avoidance & 0.98 & 0.86 & $50(84.7 \%)$ \\
\hline Score 0.00-2.99 & 3.76 & 0.54 & $9(15.3 \%)$ \\
\hline \multicolumn{4}{|l|}{ Score $3.00-5.00$} \\
\hline \multicolumn{4}{|c|}{ BUT-A Compulsive Self-monitoring } \\
\hline \multicolumn{4}{|l|}{ Score $0.00-2.99$} \\
\hline \multicolumn{4}{|l|}{ Score $3.00-5.00$} \\
\hline \multicolumn{4}{|l|}{ BUT-A Depersonalisation } \\
\hline \multicolumn{4}{|l|}{ Score $0.00-2.99$} \\
\hline Score $3.00-5.00$ & & & \\
\hline
\end{tabular}




\begin{tabular}{|c|c|c|c|}
\hline & Mean & SD & $\begin{array}{l}N(\%) \\
(n=59)\end{array}$ \\
\hline BUT-B & 14.12 & 8.92 & $59(100 \%)$ \\
\hline BUT-B Positive Symptom Total & 2.11 & 0.54 & $35(59.3 \%)$ \\
\hline Score $0-37$ & 3.67 & 0.58 & $24(40.7 \%)$ \\
\hline BUT-B Positive Symptom Distress Index & 0.31 & 0.42 & $57(96.6 \%)$ \\
\hline Score $0.00-2.99$ & 4.25 & 0.12 & $2(3.4 \%)$ \\
\hline Score $3.0-5.00$ & 0.39 & 0.56 & $57(96.6 \%)$ \\
\hline BUT-B I & 4.50 & 0.24 & $2(3.4 \%)$ \\
\hline Score $0.00-2.99$ & 1.39 & 0.90 & $38(64.4 \%)$ \\
\hline Score $3.00-5.00$ & 3.97 & 0.67 & $21(35.6 \%)$ \\
\hline BUT-B II & 0.94 & 0.86 & $51(86.4 \%)$ \\
\hline Score $0.00-2.99$ & 3.70 & 0.63 & $8(13.6 \%)$ \\
\hline Score $3.00-5.00$ & 0.93 & 0.85 & $53(89.8 \%)$ \\
\hline BUT-B III & 3.47 & 0.58 & $6(10.2 \%)$ \\
\hline Score $0.00-2.99$ & 0.48 & 0.63 & 53 (89.8\%) \\
\hline Score $3.00-5.00$ & 3.33 & 0.37 & $6(10.2 \%)$ \\
\hline BUT-B IV & 0.56 & 0.68 & 56 (94.9\%) \\
\hline Score $0.00-2.99$ & 4.83 & 0.29 & $3(5.1 \%)$ \\
\hline Score $3.00-5.00$ & 1.07 & 0.75 & 53 (89.8\%) \\
\hline BUT-B V & 3.63 & 0.41 & $6(10.2 \%)$ \\
\hline \multicolumn{4}{|l|}{ Score $0.00-2.99$} \\
\hline \multicolumn{4}{|l|}{ Score $3.00-5.00$} \\
\hline \multicolumn{4}{|l|}{ BUT-B VI } \\
\hline \multicolumn{4}{|l|}{ Score $0.00-2.99$} \\
\hline \multicolumn{4}{|l|}{ Score $3.00-5.00$} \\
\hline \multicolumn{4}{|l|}{ BUT-B VII } \\
\hline \multicolumn{4}{|l|}{ Score $0.00-2.99$} \\
\hline \multicolumn{4}{|l|}{ Score $3.00-5.00$} \\
\hline \multicolumn{4}{|l|}{ BUT-B VIII } \\
\hline \multicolumn{4}{|l|}{ Score $0.00-2.99$} \\
\hline \multicolumn{4}{|l|}{ Score $3.00-5.00$} \\
\hline SF-12 & 21.00 & 2.19 & $6(10.2 \%)$ \\
\hline Total & 34.58 & 5.43 & 53 (89.8\%) \\
\hline Score $0-24$ & 8.38 & 1.41 & $8(13.6 \%)$ \\
\hline Score $25-47$ & 14.80 & 2.68 & $51(86.4 \%)$ \\
\hline Physical Health & 12.50 & 1.85 & $8(13.6 \%)$ \\
\hline Score 0-10 & 20.33 & 3.59 & $51(86.4 \%)$ \\
\hline \multicolumn{4}{|l|}{ Score $11-20$} \\
\hline \multicolumn{4}{|l|}{ Mental Health } \\
\hline \multicolumn{4}{|l|}{ Score $0-14$} \\
\hline Score $15-27$ & & & \\
\hline
\end{tabular}




\begin{tabular}{|c|c|c|c|}
\hline & Mean & SD & $\begin{array}{l}N(\%) \\
(n=59)\end{array}$ \\
\hline BDI II & 5.00 & 2.41 & $32(54.2 \%)$ \\
\hline Score $\leq 10$ & 14.00 & 2.67 & $19(32.2 \%)$ \\
\hline Score 11-19 & 21.67 & 2.25 & $6(10.2 \%)$ \\
\hline Score $20-29$ & 34.50 & 4.95 & $2(3.4 \%)$ \\
\hline \multicolumn{4}{|l|}{ Score $\geq 30$} \\
\hline $\mathrm{BHS}$ & 1.63 & 1.10 & $32(54.2 \%)$ \\
\hline Score 0-4 & 4.87 & 1.18 & $23(39.0 \%)$ \\
\hline Score 5-9 & 11.25 & 1.89 & $4(6.8 \%)$ \\
\hline Score $10-14$ & 0.00 & 0.00 & $0(0 \%)$ \\
\hline Score 15-20 & & & \\
\hline
\end{tabular}

The correlational analyses (Pearson's r) among the weight, BMI and BUT with the psychological outcomes (SCL-90-R, SF-12, BDI II and BHS) are reported in Table 2. 
Table 2

Correlational analyses (Pearson's r) among the weight, BMI and BUT with SCL-90-R, SF-

\begin{tabular}{|c|c|c|c|c|c|c|c|c|c|c|}
\hline & $\begin{array}{l}\text { SCL- } \\
\text { 90-R } \\
\text { Global } \\
\text { Severity } \\
\text { Index }\end{array}$ & $\begin{array}{l}\text { SCL-90-R } \\
\text { Positive } \\
\text { Symptoms } \\
\text { Total }\end{array}$ & $\begin{array}{l}\text { SCL-90-R } \\
\text { Positive } \\
\text { Symptom } \\
\text { Distress } \\
\text { Index }\end{array}$ & $\begin{array}{l}\text { SCL-90-R } \\
\text { Somatisation }\end{array}$ & $\begin{array}{l}\text { SCL-90-R } \\
\text { Obsessiveness- } \\
\text { Compulsiveness }\end{array}$ & $\begin{array}{l}\text { SCL-90-R } \\
\text { Interpersonal } \\
\text { Hypersensitivity }\end{array}$ & $\begin{array}{l}\text { SCL-90-R } \\
\text { Depression }\end{array}$ & $\begin{array}{l}\text { SCL- } \\
\text { 90-R } \\
\text { Anxiety }\end{array}$ & $\begin{array}{l}\text { SCL-90- } \\
\text { R } \\
\text { Hostility }\end{array}$ & $\begin{array}{l}\mathrm{SC} \\
9 \mathrm{C} \\
\mathrm{Pr} \\
\mathrm{Ar}\end{array}$ \\
\hline \multirow[t]{2}{*}{ Weight } & $r=-.19$ & $r=-.083$ & $r=-.20$ & $r=-.28$ & $r=-.21$ & $r=-.11$ & $r=-.14$ & $r=-.15$ & $r=-.05$ & $r=$ \\
\hline & $p=.151$ & $p=.533$ & $p=.123$ & $p=.031$ & $p=.115$ & $p=.406$ & $p=.285$ & $p=.253$ & $p=.679$ & $p=$ \\
\hline \multirow[t]{2}{*}{ BMI } & $r=-29$ & $r=-.21$ & $r=-.22$ & $r=-.35$ & $r=-.32$ & $r=-.18$ & $r=-.27$ & $r=-.23$ & $r=-.16$ & -.0 \\
\hline & $p=.027$ & $p=.101$ & $p=.089$ & $p=.006$ & $p=.014$ & $p=.179$ & $p=.038$ & $p=.073$ & $p=.220$ & $p=$ \\
\hline \multirow{2}{*}{$\begin{array}{l}\text { BUT-A Global } \\
\text { Severity Index }\end{array}$} & $r=.73$ & $r=.64$ & $r=.58$ & $r=.51$ & $r=.62$ & $r=.69$ & $r=.68$ & $r=.60$ & $r=.60$ & $r=$ \\
\hline & $\mathrm{p}<.001$ & $p=<.001$ & $p=<.001$ & $p=<.001$ & $p=<.001$ & $p=<.001$ & $\mathrm{p}=<.001$ & $\begin{array}{l}\mathrm{p}= \\
<.001\end{array}$ & $\mathrm{p}<.001$ & $\begin{array}{l}\mathrm{p}= \\
<. \mathrm{c}\end{array}$ \\
\hline \multirow{2}{*}{$\begin{array}{l}\text { BUT-A Weight } \\
\text { Phobia }\end{array}$} & $r=.63$ & $r=.56$ & $r=.51$ & $r=.40$ & $r=.51$ & $r=.59$ & $r=.58$ & $r=.51$ & $r=.56$ & $r=$ \\
\hline & $\begin{array}{l}\mathrm{p}= \\
<.001\end{array}$ & $p=<.001$ & $p=<.001$ & $p=<.001$ & $p=<.001$ & $p=<.001$ & $\mathrm{p}=<.001$ & $\begin{array}{l}\mathrm{p}= \\
<.001\end{array}$ & $p=<.001$ & $\begin{array}{l}\mathrm{p}= \\
<. \mathrm{c}\end{array}$ \\
\hline \multirow{2}{*}{$\begin{array}{l}\text { BUT-A Body } \\
\text { Image Concern }\end{array}$} & $r=.59$ & $r=.52$ & $r=.51$ & $r=.38$ & $r=.47$ & $r=.58$ & $r=.57$ & $r=.49$ & $r=.46$ & $r=$ \\
\hline & $\begin{array}{l}\mathrm{p}= \\
<.001\end{array}$ & $p=<.001$ & $p=<.001$ & $p=.003$ & $p=<.001$ & $p=<.001$ & $p=<.001$ & $\begin{array}{l}\mathrm{p}= \\
<.001\end{array}$ & $p=<.001$ & $\begin{array}{l}\mathrm{p}= \\
<. \mathrm{c}\end{array}$ \\
\hline \multirow[t]{2}{*}{ BUT-A Avoidance } & $r=.73$ & $r=.65$ & $r=.52$ & $r=.53$ & $r=.59$ & $r=.67$ & $r=.69$ & $r=.61$ & $r=.64$ & $r=$. \\
\hline & $\begin{array}{l}\mathrm{p}= \\
<.001\end{array}$ & $p=<.001$ & $\mathrm{p}=<.001$ & $p=<.001$ & $p=<.001$ & $p=<.001$ & $\mathrm{p}=<.001$ & $\begin{array}{l}\mathrm{p}= \\
<.001\end{array}$ & $p=<.001$ & $\begin{array}{l}\mathrm{p}= \\
<. \mathrm{c}\end{array}$ \\
\hline \multirow{2}{*}{$\begin{array}{l}\text { BUT-A } \\
\text { Compulsive Self- } \\
\text { monitoring }\end{array}$} & $r=.53$ & $r=.42$ & $r=.52$ & $r=.42$ & $r=.53$ & $r=.55$ & $r=.42$ & $r=.35$ & $r=.46$ & $r=$. \\
\hline & $\begin{array}{l}\mathrm{p}= \\
<.001\end{array}$ & $p=.001$ & $\mathrm{p}=<.001$ & $\mathrm{p}=.001$ & $\mathrm{p}=<.001$ & $\mathrm{p}=<.001$ & $p=.001$ & $p=.006$ & $\mathrm{p}=<.001$ & $p=$ \\
\hline \multirow[t]{2}{*}{$\begin{array}{l}\text { BUT-A } \\
\text { Depersonalisation }\end{array}$} & $r=.69$ & $r=.64$ & $r=.45$ & $r=.51$ & $r=.63$ & $r=.60$ & .66 & $r=.62$ & $r=.50$ & $r=$. \\
\hline & $\begin{array}{l}\mathrm{p}= \\
<.001\end{array}$ & $\mathrm{p}=<.001$ & $\mathrm{p}=<.001$ & $\mathrm{p}=<.001$ & $p=<.001$ & $p=<.001$ & $\mathrm{p}=<.001$ & $\begin{array}{l}\mathrm{p}= \\
<.001\end{array}$ & $p=<.001$ & $\begin{array}{l}\mathrm{p}= \\
<. \mathrm{c}\end{array}$ \\
\hline \multirow{2}{*}{$\begin{array}{l}\text { BUT-B Positive } \\
\text { Symptom Total }\end{array}$} & $r=.61$ & $r=.56$ & $r=.65$ & $r=.52$ & $r=.66$ & $r=.48$ & $r=.49$ & $r=.55$ & $r=.59$ & $r=$. \\
\hline & $\begin{array}{l}\mathrm{p}= \\
<.001\end{array}$ & $p=<.001$ & $p=.008$ & $p=<.001$ & $\mathrm{p}=<.001$ & $\mathrm{p}=<.001$ & $p=<.001$ & $\begin{array}{l}\mathrm{p}= \\
<.001\end{array}$ & $\mathrm{p}=<.001$ & $p=$ \\
\hline \multirow{2}{*}{$\begin{array}{l}\text { BUT-B Positive } \\
\text { Symptom } \\
\text { Distress Index }\end{array}$} & $r=.42$ & .29 & .45 & $r=.28$ & $r=.48$ & $r=.32$ & $r=.34$ & $r=.35$ & $r=.27$ & $r=$. \\
\hline & $p=.001$ & $p=.026$ & $p=<.001$ & $p=.030$ & $p=<.001$ & $p=.013$ & $p=.008$ & $p=.007$ & $p=.035$ & $p=$ \\
\hline \multirow[t]{2}{*}{ BUT-B I } & $r=.52$ & .39 & .39 & .30 & $r=.67$ & $r=.36$ & $r=.3715$ & .47 & $r=.67$ & $r=$ \\
\hline & $\begin{array}{l}\mathrm{p}= \\
<.001\end{array}$ & $p=.002$ & $\mathrm{p}=<.001$ & $\mathrm{p}=.020$ & $\mathrm{p}=<.001$ & $p=.005$ & $p=.004$ & $\begin{array}{l}\mathrm{p}= \\
<.001\end{array}$ & $\mathrm{p}=<.001$ & $p=$ \\
\hline \multirow[t]{2}{*}{ BUT-B II } & $r=.56$ & $r=.36$ & $r=.49$ & $r=.34$ & $r=.71$ & $r=.42$ & $r=.39$ & $r=.47$ & $r=.72$ & $r=$. \\
\hline & $\begin{array}{l}\mathrm{p}= \\
<.001\end{array}$ & $p=.005$ & $p=<.001$ & $p=.008$ & $p=<.001$ & $p=.001$ & $p=.002$ & $\begin{array}{l}\mathrm{p}= \\
<.001\end{array}$ & $\mathrm{p}=<.001$ & $p=$ \\
\hline \multirow[t]{2}{*}{ BUT-B III } & $r=.55$ & $r=.53$ & $r=.42$ & $r=.54$ & $r=.50$ & .42 & $r=.50$ & $r=.5254$ & $r=.32$ & $r=$. \\
\hline & $\begin{array}{l}\mathrm{p}= \\
<.001\end{array}$ & $\mathrm{p}=<.001$ & $p=.001$ & $\mathrm{p}=<.001$ & $p=<.001$ & $p=.001$ & $p=<.001$ & $\begin{array}{l}\mathrm{p}= \\
<.001\end{array}$ & $p=.014$ & $p=$ \\
\hline \multirow[t]{2}{*}{ BUT-B IV } & $r=.64$ & $r=.54$ & $r=.54$ & $r=.55$ & $r=.72$ & $r=.47$ & $r=.51$ & $r=.58$ & $r=.50$ & $r=$. \\
\hline & $\begin{array}{l}\mathrm{p}= \\
<.001\end{array}$ & $p=<.001$ & $p=<.001$ & $p=<.001$ & $p=<.001$ & $p=<.001$ & $\mathrm{p}=<.001$ & $\begin{array}{l}\mathrm{p}= \\
<.001\end{array}$ & $\mathrm{p}=<.001$ & $p=$ \\
\hline \multirow[t]{2}{*}{ BUT-B V } & $r=.67$ & $r=.56$ & $r=.50$ & $r=.65$ & $r=.69$ & $r=.51$ & $r=.54$ & $r=.60$ & $r=.58$ & $r=$ \\
\hline & $\begin{array}{l}\mathrm{p}= \\
<.001\end{array}$ & $p=<.001$ & $\mathrm{p}=<.001$ & $p=<.001$ & $p=<.001$ & $p=<.001$ & $\mathrm{p}=<.001$ & $\begin{array}{l}\mathrm{p}= \\
<.001\end{array}$ & $\mathrm{p}=<.001$ & $\begin{array}{l}\mathrm{p}= \\
<. \mathrm{c}\end{array}$ \\
\hline \multirow[t]{2}{*}{ BUT-B VI } & $r=.31$ & $r=.21$ & $r=.29$ & $r=.16$ & $r=.44$ & $r=.28$ & $r=.19$ & $r=.29$ & $r=.40$ & $r=$. \\
\hline & $p=.017$ & $p=.112$ & $p=.026$ & $p=.227$ & $p=.001$ & $p=.033$ & $p=.146$ & $p=.024$ & $p=.001$ & $p=$ \\
\hline
\end{tabular}




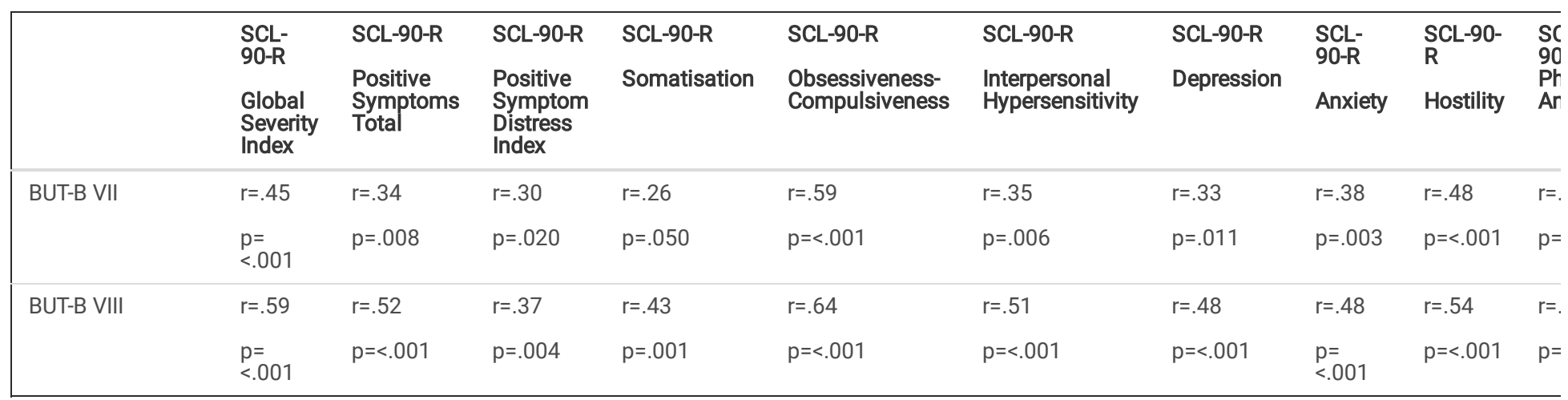

Weight was only negatively correlated with SCL-90-R subscale of somatisation, while BMI was negatively correlated with Global Severity Index of SCL-90-R, and SCL-90-R subscales of somatisation, obsessiveness-compulsiveness, and depression (see Table 2).

BUT-A Global Severity Index and most of its subscales were positively correlated with all SCL-90-R scales, BDI II and BHS and was negatively correlated with SF-12 scales (see Table 2). Also, BUT-B Positive Symptom Distress Index, similarly to the BUT-B Positive Symptom Total, was positively correlated with all SCL90-R scale, and BDI II, however it did not show significant correlation with BHS. Moreover BUT-B Positive Symptom Total was negatively correlated with SF-12 scales (see Table 2).

The multiple regression analyses performed on the psychological outcomes (SCL-90-R Global Severity Index, SCL-90-R Positive Symptom Total, SF-12 Physic Health, SF-12 Mental Health, BDI II, and BHS) with BMI, BUT-A Global Severity Index and BUT-B Positive Symptom Distress Index as predictors showed the following results. As regard to the SCL-90-R Global Severity Index, the regression model was significant, and the BUT-A Global Severity Index was a significant predictor (see Figure 1). As regard to the SCL-90-R Positive Symptom Total, the regression model was significant, and the BUT-A Global Severity Index was a significant predictor (see Figure 1). As regard to the SF-12 Physical Health, the regression model was not significant (see Figure 1). Instead, as regard to the SF-12 mental Health, the regression model was significant, and the BUT-A Global Severity Index was a significant predictor (see Figure 1). As regard to the BDI II, the regression model was significant, and the BUT-A Global Severity Index was a significant predictor (see Figure 1). Finally, as regard to the BHS, the regression model was significant, and the BUT-A Global Severity Index was a significant predictor (see Figure 1).

\section{Discussion}

This study investigated the association between the perception of one's own body image in relation to aspects of psychophysical health in a preliminary phase of bariatric surgery.

The main finding was that a higher dissatisfaction with the perception of one's own body image was strongly associated with higher psychological symptoms and a lower psychological health. This finding is consistent with the result of a descriptive study that has investigated this kind of positive and direct relationship between the body image and the psychological well-being [26]. A higher satisfaction of one's own body image corresponds to a greater and better psychological health. This result could be explained by the fact that, due to the multidimensional and complexity of body image, there may be several factors that influence the development of a negative body image, mainly in people suffering from obesity [27]. These findings seem to support that the perception of one's own body image is not only dependent on a possible mismatch between perceived and desired body shapes, but also on the presence of mental images, emotions and thoughts that lead to associate body appearance with personal value in the social context [14]. Indeed, these people are often made fun of for their weight and appearance, the social stigma and discrimination can worse low self-esteem and depression [28].

Another finding of the present study was that the BMI did not show any significant association with the psychopathological outcome and with quality of life. This result suggests that the effective real physical weight did not represent a risk factor per se. In fact, despite previous studies on obese patients showed strong association between the BMI and the psychopathological state [29-31], the present study showed only a few correlations between BMI and some psychological variables. Moreover, in the regression models the BMl, inserted as predictor with the two body image measures, did not show any significant effect on the psychological outcome. These findings suggest a greater relevance of the body image on the psychological state compared to the BMI. A possible explanation of this result seems to be that the experience of one's own weight is majorly related to the expectations that the individual and the others have about body image more than to the objective weight with important clinical implications. This perspective motivates the consideration of body image as a crucial variable in the outcome of interventions focused for the treatment of obesity.

In the present study, only the dissatisfaction for body image was associated with psychological outcomes (BUT- A), while the hate for a specific part of one's own body (BUT- B) showed lower grades of correlation with the psychological outcomes and when inserted in the regression models, it did not show any significant effects. This result suggests that the preoccupation for specific parts of one's own body seems to have less relevant role compared to the global dissatisfaction for body image in the relationship with the psychological outcomes.

In contrast with previous studies $[10,11]$, the present study did not show high presence of hopelessness in the obese sample. This result could be explained by the fact that the participants were waiting for a bariatric surgery to solve their obesity. This expectation could influence their feelings of hopelessness. It seems relevant to investigate the evolution of hopelessness after the bariatric surgery to identify risk factors in its increase.

Finally, the participants in our study, obese patients undergoing bariatric surgery, showed well-defined psychological aspects in the pre-operative phase. They reported moderate and severe levels of psychological distress, especially in the somatic, obsessive-compulsive, interpersonal hypersensitivity, and depressive

Page $10 / 13$ 
aspects. Furthermore, regarding the perception of their own body image, the participants showed a high level of discomfort, particularly on aspects related to weight phobia and preoccupation with their body image. Finally, they reported low levels of both physical and mental well-being and a mild presence of hopelessness feelings.

This is the first study that investigated the relative role of body image compared to body mass index in the association with psychological outcome in obese patients.

This study had some limitations. The first was that self-report measures were used. The second limitation was found to be the lack of gender balance in the participant group. Finally, the sample was recruited from a single health-care centre.

\section{What is already known on this subject?}

The body image dissatisfaction is more common in people with severe obesity, particularly those who choose bariatric surgery to cure their obesity.

\section{What does this study add?}

The present study suggests a more relevant role of body image compared to the BMI in the association with psychological outcomes.

\section{References}

1. De Luca M, Angrisani L, Himpens J, Busetto L, Scopinaro N, Weiner R, Sartori A, Stier C, Lakdawala M, Bhasker AG, Buchwald H, Dixon J, Chiappetta S, Kolberg HC, Frühbeck G, Sarwer DB, Suter M, Soricelli E, Blüher M, Shikora S (2016) Indications for surgery for obesity and weight-related diseases: Position statements from the international federation for the surgery of obesity and metabolic disorders (IFSO). Obesity Surgery 26(8):16591696. https://doi.org/10.1007/s11695-016-2271-4

2. Dalle Grave R, Cuzzolaro M, Calugi S, Tomasi F, Temperilli F, Marchesini G, QUOVADIS Study Group (2007) The effect of obesity management on body image in patients seeking treatment at medical centers. Obesity (Silver Spring, Md.) 15(9), 2320-2327. https://doi.org/10.1038/oby.2007.275

3. Sarwer DB, Thompson J K, Cash TF (2005) Body image and obesity in adulthood. The Psychiatric clinics of North America 28(1), 69VIII. https://doi.org/10.1016/j.psc.2004.09.002

4. Husky MM, Mazure CM, Ruffault A, Flahault C, Kovess-Masfety V (2018) Differential Associations Between Excess Body Weight and Psychiatric Disorders in Men and Women. Journal of women's health, 27(2):183-190. https://doi.org/10.1089/jwh.2016.6248

5. Lai C, Aceto P, Santucci F R, Pierro L, Petrucci I, Cacioppo M, Castelnuovo G, Sollazzi L, Bellantone R, Raffaelli M (2021). Preoperative psychological characteristics affecting mid-term outcome after bariatric surgery: a follow-up study. EWD 26(2):585-590. https://doi.org/10.1007/s40519-020-00892-w

6. Paone E, Pierro L, Damico A, Aceto P, Campanile FC, Silecchia G, Lai C (2019) Alexithymia and weight loss in obese patients underwent laparoscopic sleeve gastrectomy. EWD, 24(1):129-134. https://doi.org/10.1007/s40519-017-0381-1

7. Richard A, Rohrmann S, Lohse T, Eichholzer M (2016) Is body weight dissatisfaction a predictor of depression independent of body mass index, sex and age? Results of a cross-sectional study. BMC Public Health 16:863. https://doi.org/10.1186/s12889-016-3497-8

8. Soulliard ZA, Brode C, Tabone LE, Abunannaja S, Szoka N, Cox S (2021) The role of body appreciation, weight bias internalization, and disordered eating behaviors among presurgical bariatric patients. Surgery for obesity and related diseases: official journal of the American Society for Bariatric Surgery 17(5):1000-1007. https://doi.org/10.1016/j.soard.2020.12.012

9. deMeireles AJ, Carlin AM, Bonham AJ, Cassidy R, Ross R, Stricklen A, Finks J, Ghaferi AA (2020) A Longitudinal Analysis of Variation in Psychological Well-being and Body Image in Patients Before and After Bariatric Surgery. Annals of surgery 271(5):885890. https://doi.org/10.1097/SLA.0000000000003146

10. Felske AN, Williamson TM, Scurrey S, Telfer JA, Campbell TS, Rash JA (2021) The Influence of Weight-Related Self-Esteem and Symptoms of Depression on Shape and Weight Concerns and Weight-Loss 12 Months After Bariatric Surgery. Obesity surgery 31(3):1062-1072. https://doi.org/10.1007/s11695020-05097-9

11. Perdue TO, Schreier A, Neil J, Carels R, Swanson M (2020) A Concept Analysis of Disturbed Body Image in Bariatric Surgery Patients. International journal of nursing knowledge 31(1):74-81. https://doi.org/10.1111/2047-3095.12220

12. Sarwer DB, Wadden TA, Moore RH, Eisenberg MH, Raper SE, Williams NN (2010) Changes in quality of life and body image after gastric bypass surgery. Surgery for obesity and related diseases: official journal of the American Society for Bariatric Surgery 6(6):608614. https://doi.org/10.1016/j.soard.2010.07.015

13. Song P, Patel NB., Gunther S, Li CS, Liu Y, Lee CY, Kludt NA, Patel KB, Ali MR, Wong MS (2016) Body Image \& Quality of Life: Changes with Gastric Bypass and Body Contouring. Annals of plastic surgery, 76 Suppl 3(Suppl 3), S216-S221. https://doi.org/10.1097/SAP.0000000000000788

14. Mento C, Silvestri M C, Muscatello M, Rizzo A, Celebre L, Cedro C, Zoccali RA, Navarra G, Bruno A (2021) The role of body image in obese identity changes post bariatric surgery. EWD. https://doi.org/10.1007/s40519-021-01270-w

15. Pompili M, Girardi P, Innamorati M, Tatarelli G, Ruberto A, Ferrari V, Tatarelli R (2007) Body uneasiness and suicide risk in a non-clinical sample of university students. Archives of suicide research: official journal of the International Academy for Suicide Research 11(2):193202. https://doi.org/10.1080/13811110600896392

16. Fried M, Yumuk V, Oppert JM, Scopinaro N, Torres A, Weiner R, Yashkov Y, Frühbeck G, International Federation for Surgery of Obesity and Metabolic Disorders-European Chapter (IFSO-EC), European Association for the Study of Obesity (EASO), European Association for the Study of Obesity Obesity 
Management Task Force (EASO OMTF) (2014) Interdisciplinary European guidelines on metabolic and bariatric surgery. Obes Surg 24(1):4255. https://doi.org/10.1007/s11695-013-1079-8

17. Derogatis LR, Lipman RS, Covi L (1973) SCL-90: an outpatient psychiatric rating scale-preliminary report. Psychopharmacology bulletin, 9(1):13-28.

18. Prunas A, Sarno I, Preti E, Madeddu F, Perugini M (2012) Psychometric properties of the Italian version of the SCL-90-R: a study on a large community sample. European psychiatry: the journal of the Association of European Psychiatrists 27(8):591-597. https://doi.org/10.1016/j.eurpsy.2010.12.006

19. Cuzzolaro M, Vetrone G, Marano G, Garfinkel PE (2006) The Body Uneasiness Test (BUT): development and validation of a new body image assessment scale. EWD 11(1):1-13. https://doi.org/10.1007/BF03327738

20. Ware JJr, Kosinski M, Keller SD (1996) A 12-Item Short-Form Health Survey: construction of scales and preliminary tests of reliability and validity. Medical care 34(3):220-233. https://doi.org/10.1097/00005650-199603000-00003

21. Apolone G, Mosconi P (1998) The Italian SF-36 Health Survey: translation, validation and norming. Journal of clinical epidemiology 51(11):10251036. https://doi.org/10.1016/s0895-4356(98)00094-8

22. Beck AT, Steer RA, Ball R, Ranieri W (1996) Comparison of Beck Depression Inventories -IA and -II in psychiatric outpatients. Journal of personality assessment 67(3):588-597. https://doi.org/10.1207/s15327752jpa6703_13

23. Sica C, Ghisi M (2007) The Italian versions of the Beck Anxiety Inventory and the Beck Depression Inventory-II: Psychometric properties and discriminant power. In Leading-Edge Psychological Tests and Testing Research, pp 27-50.

24. Beck AT, Weissman A, Lester D, Trexler L (1974) The measurement of pessimism: the hopelessness scale. Journal of consulting and clinical psychology 42(6):861-865. https://doi.org/10.1037/h0037562

25. Pompili M, lliceto P, Lester D, Innamorati M, Girardi P, Tatarelli R (2009a) BHS Beck Hopelessness Scale: manuale. Giunti OS, Firenze.

26. Dotse JE, Asumeng M (2015) Relationship between Body Image Satisfaction and Psychological Well-Being: The Impact of Afrocentric Values. Journal of Social Science Studies 2:320-42. https://doi.org/10.5296/jsss.v2i1.6843

27. Cash TF (2004) Body image: past, present, and future. Body image 1(1):1-5. https://doi.org/10.1016/S1740-1445(03)00011-1

28. Latner JD, Wilson RE (2011) Obesity and body image in adulthood. In: Cash TF, Smolak L, editors.Body image: a handbook of science, practice, and prevention. Guilford Press, New York pp 189-198.

29. Carpenter KM, Hasin DS, Allison DB, Faith MS (2000) Relationships between obesity and DSM-IV major depressive disorder, suicide ideation, and suicide attempts: results from a general population study. American journal of public health 90(2):251-257. https://doi.org/10.2105/ajph.90.2.251

30. Ford ES, Moriarty DG, Zack MM, Mokdad AH Chapman DP (2001) Self-reported body mass index and health-related quality of life: findings from the Behavioral Risk Factor Surveillance System. Obesity research 9(1):21-31. https://doi.org/10.1038/oby.2001.4

31. Robertson S, Davies M, Winefield H (2015) Why weight for happiness? Correlates of BMI and SWB in Australia. Obesity research \& clinical practice 9(6):609-612. https://doi.org/10.1016/j.orcp.2015.04.011

\section{Figures}




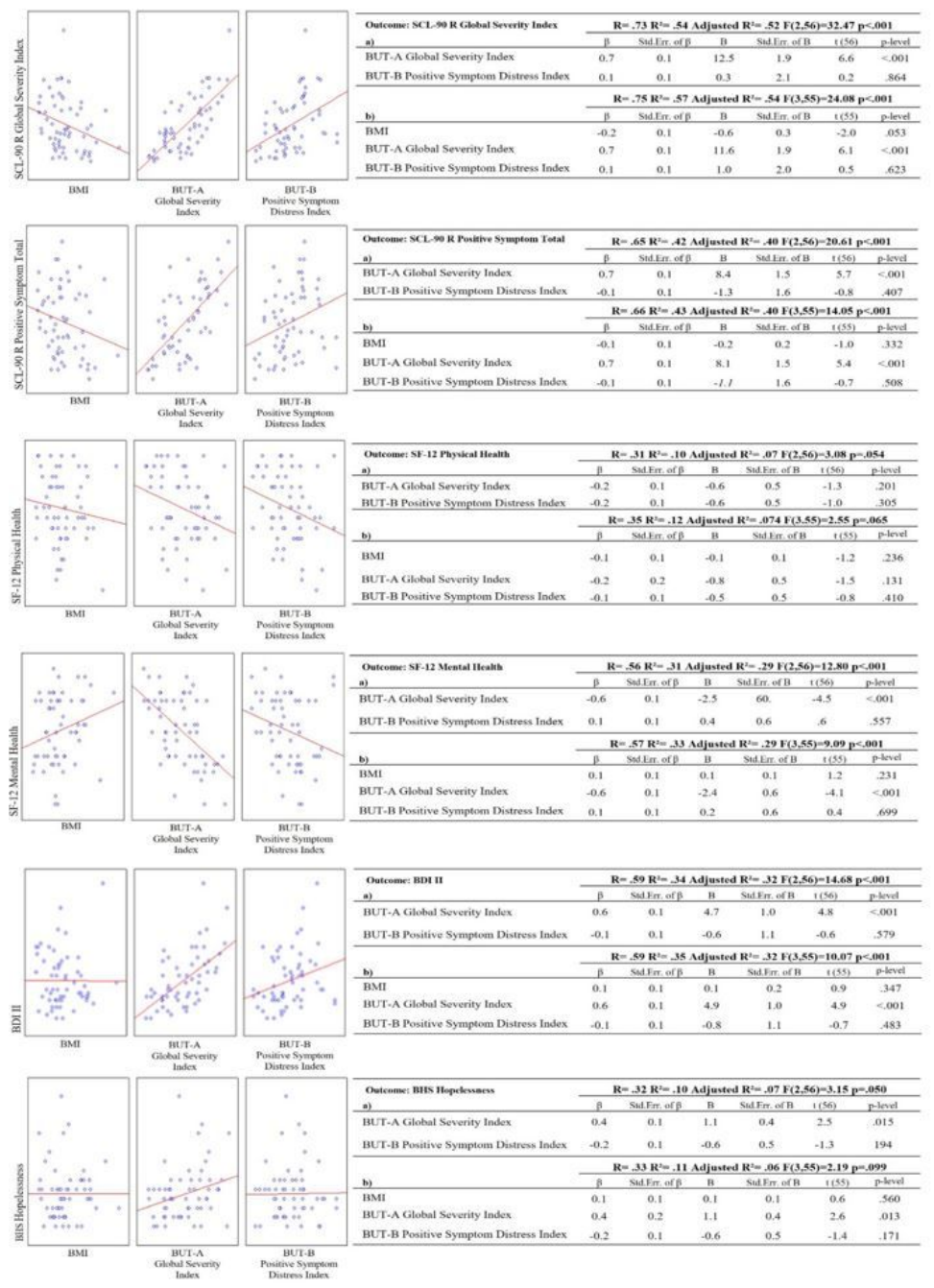

\section{Figure 1}

Regression Lines and Multiple Regression Model with a) BUT-A Global Severity Index, BUT-B Positive Symptom Distress Index, and b) BMI, BUT-A Global Severity Index, BUT-B Positive Symptom Distress Index as predictors of SCL-90 Global Severity Index, SCL-90 R Positive Symptom Total, Sf-12 Physical Health, SF-12 Mental Health, BDI II, and BHS. 